\title{
EFFECTIVENESS OF SOME EXTENSION METHODS IN TRANSFERRING EXTENSION RECOMMENDATIONS FOR RURAL WOMEN IN FAMILY HEALTH AREA “A FIELD STUDY IN DAKAHALIA GOVERNORATE.
}

El- Gamal, M. M. A. ; M. A. M Abd El-Magieed and A. M. A. RAMADAN Agric. Extension and Rural Society, Fac. of Agric., Mans. Univ.

فعالية بعض الطرق الإرشادية في نقل التوصيات الإرشادية للمرأه الريفية في مجال

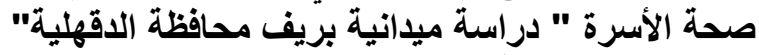

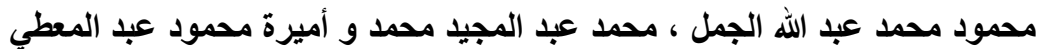

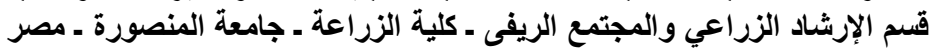

الملخص

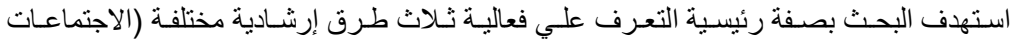

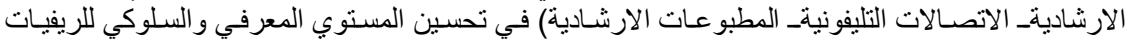

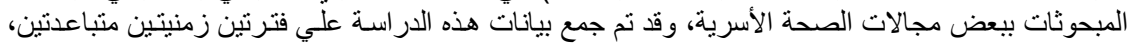

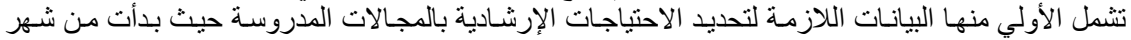

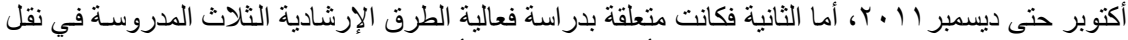

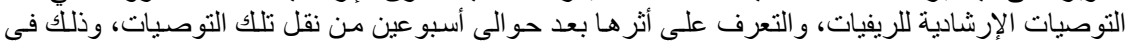

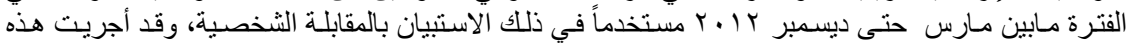

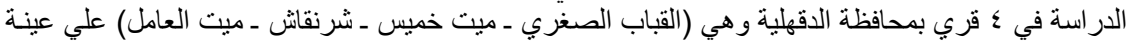

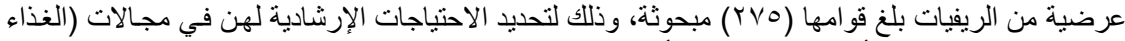

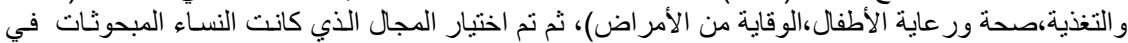

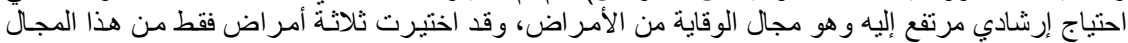

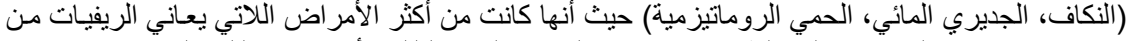

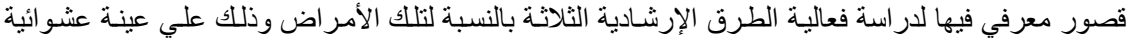

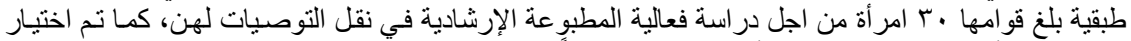

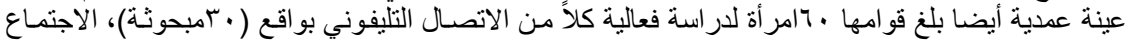

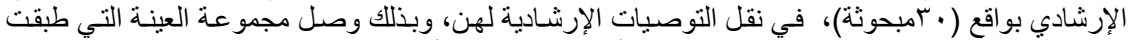

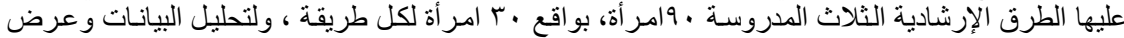

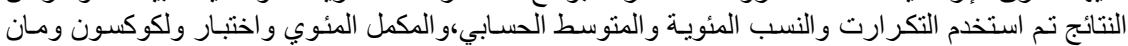

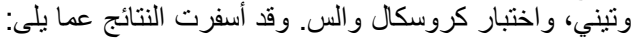

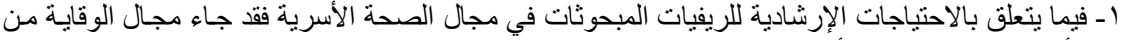

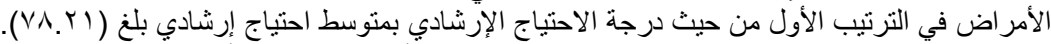

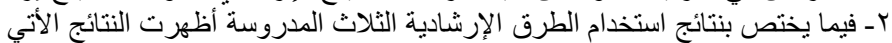

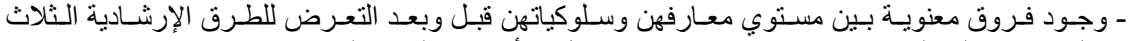

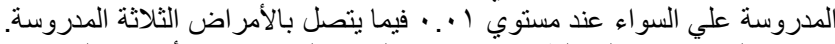

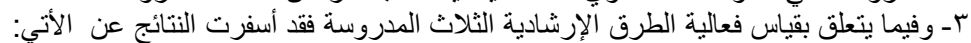

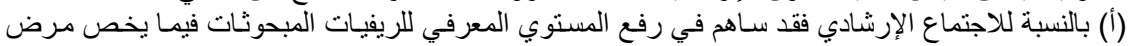

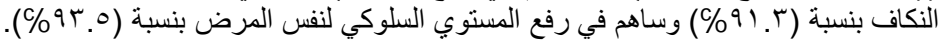

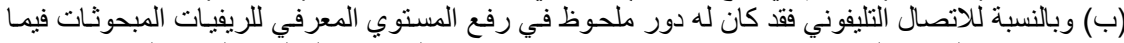

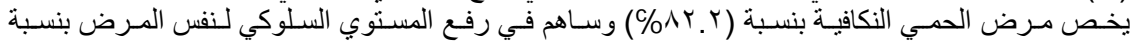




\section{El- Gamal, M. M. A. et al.}

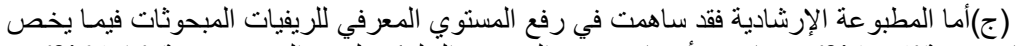

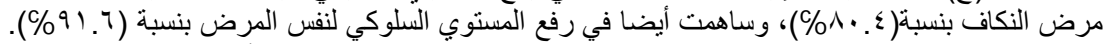

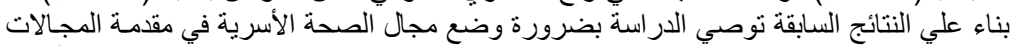

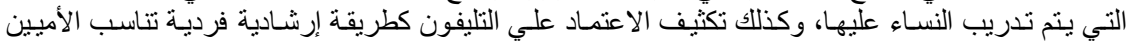
و المتعلمين ، و عدم النظر إليه علي أنه طريقة تدفع لنجاح الطرق الإرشادية النّاد الأخرى فقط.

\section{المقدمة ومثكلة البحث}

يعتبر وضع المر أة ومساهمتها في التنمية في أي مجتمع أحد المعايير الأساسبة لقياس درجـة تقدمها،

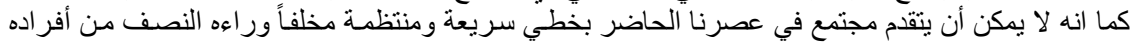

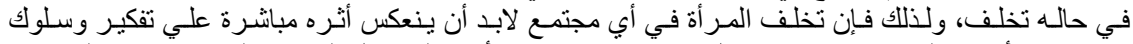

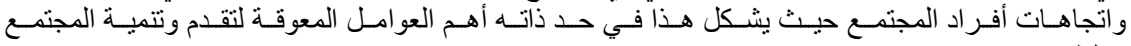

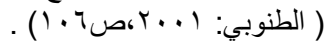

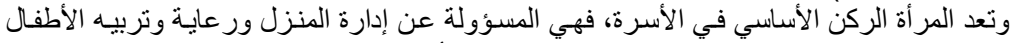

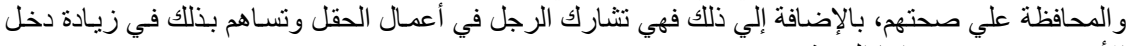
الأسرة وتحسين مستو الها المعيشي. بالإفيافي.

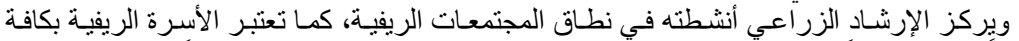

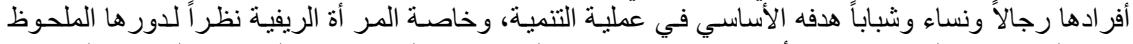

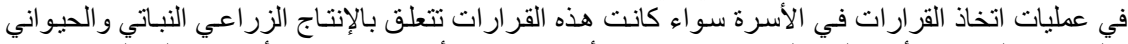

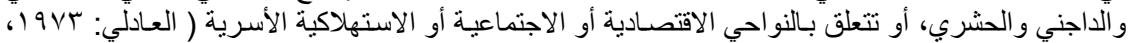

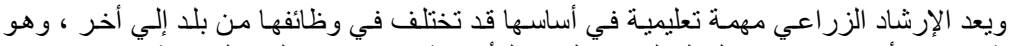

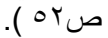

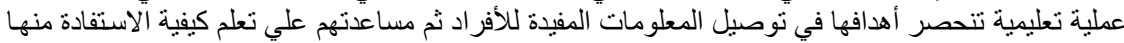

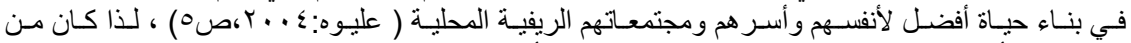

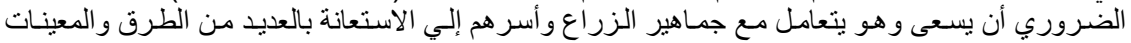

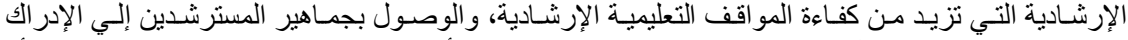

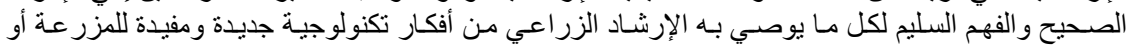

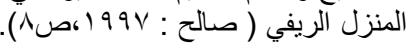

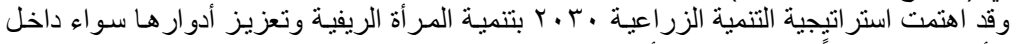

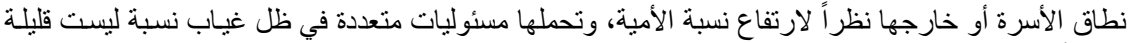

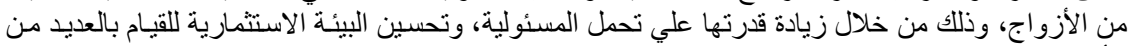

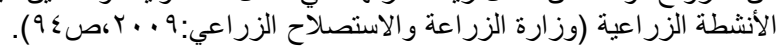

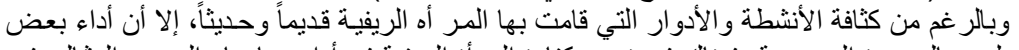

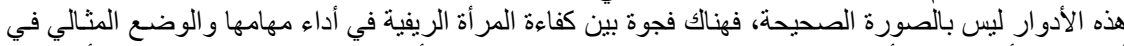

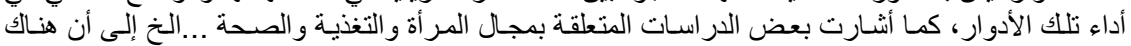

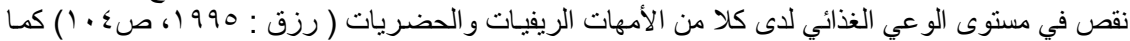

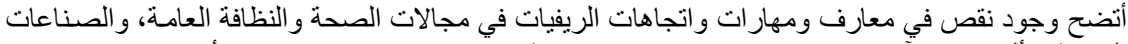

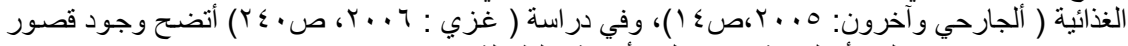

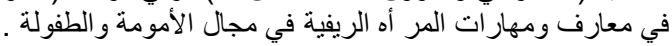

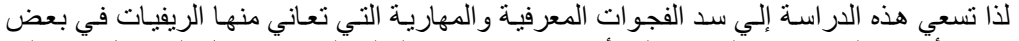

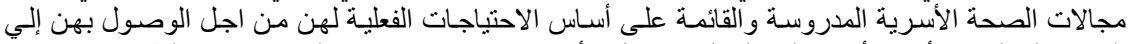

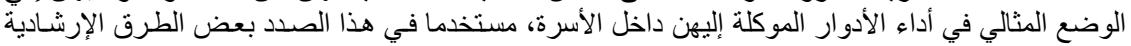

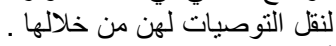
أهداف البحث

يستهدف هذا البحث بصفة رئيسية تحقيق الأهداف التالية:

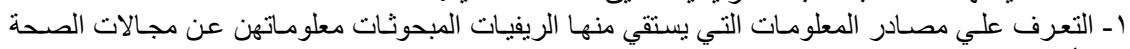


r- التعرف على الاحتياجات الإرشادية للريفيات المبحوثات في بعض مجالات الإنات الصحة الأسرية المدروسة

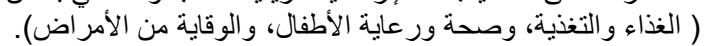

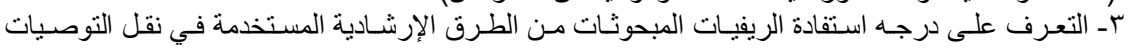
الإرشادية إليهن.

عـ التعرف علي الثر الطرق الإرشادية الثلاث المدروسة (المطبو عـة الارشـاديةـ الاجتمـاع الارشـاديـ الاتصـال

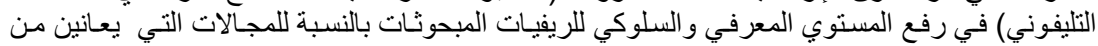

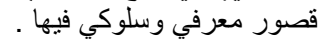
هـ قياس فعالية الطرق الإرشادية المدروسة في نقل التوصيات الإرشادية للريفيات في المجالات اللاتي يعانين من قصور معرفي فيها.

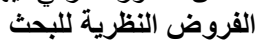

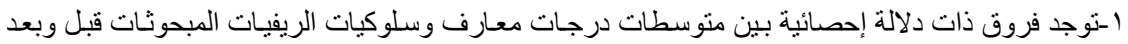

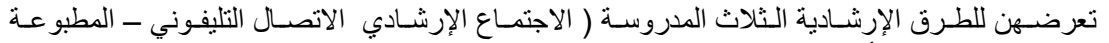

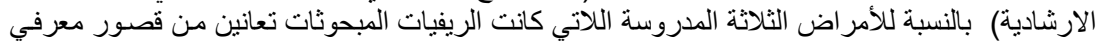

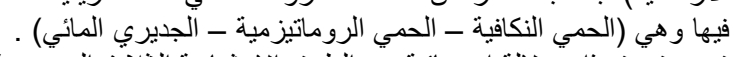

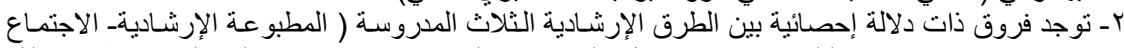

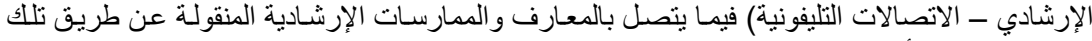

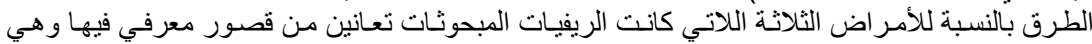

(الحمي النكافية ـ الحمي الروماتيزمية ــ الجديري المائي).

\section{الطريقة البحثية}

أولاً: التعريفات الإجرائية

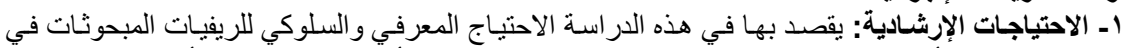

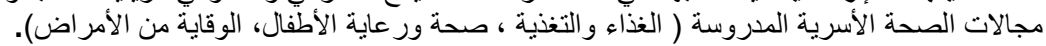

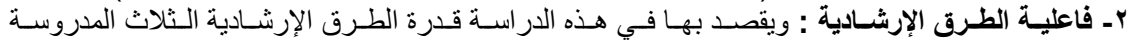

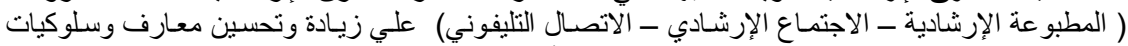

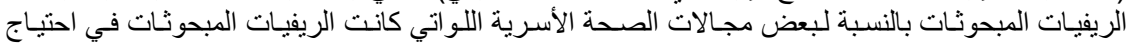
إرشادي إليها.

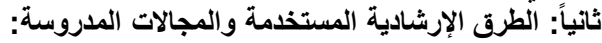

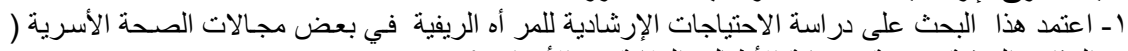

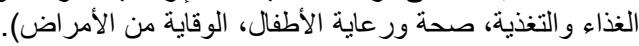

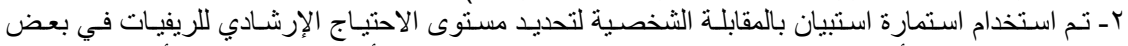

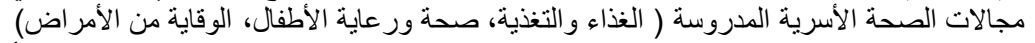

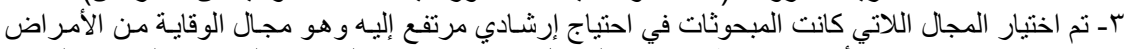

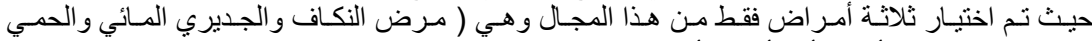
الروماتيزمية ) علي أساس أنها أكثر الأمر اض اللاتي كن تعانين قصور مُ معرف في فيها وليس سلوكي للسبيين التاليين: أـ لان الئعارف هي أساس السلوك وهي التي تؤدي إلي تغير السلوك فيما بعد.

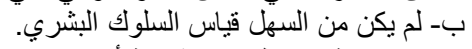

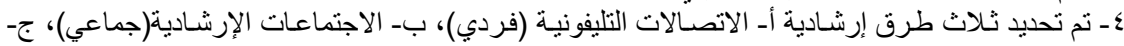

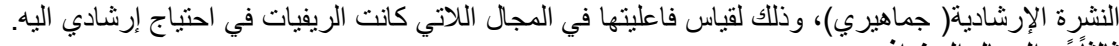

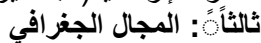

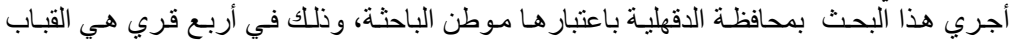

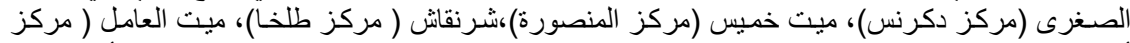

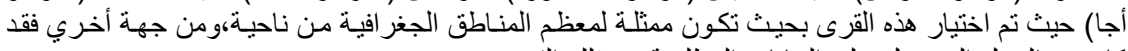

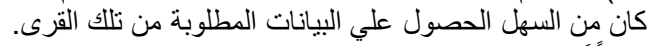
رابعاًُ: المجال البشرى البطر 


\section{El- Gamal, M. M. A. et al.}

تم اختيار عينة عرضية بلغ قوامها بV امر أة ريفية من إجمالي عدد الأسر الريفية بالقرى الأربعة

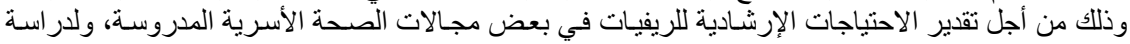

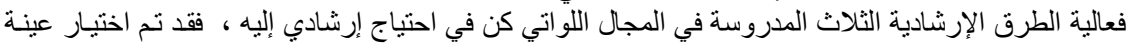

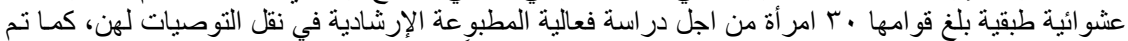

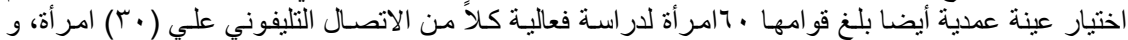

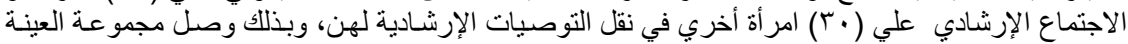

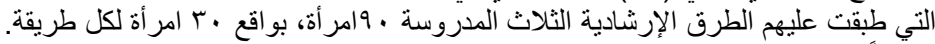

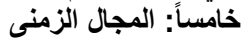

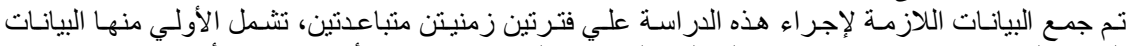

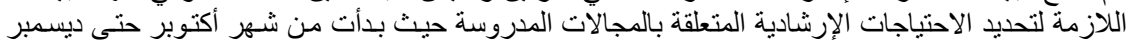

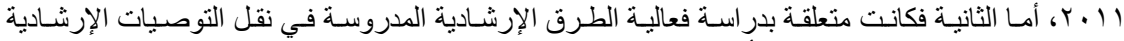

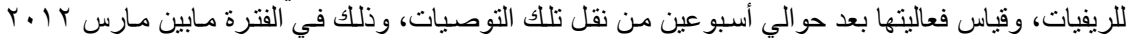

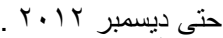
سادساً:ً: المعالجة الكمية للبيانات

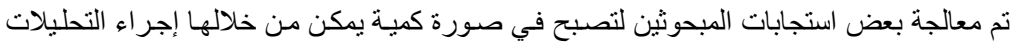

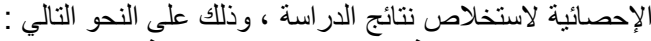

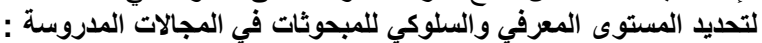

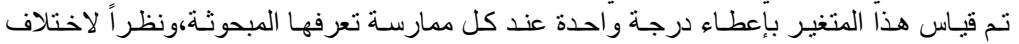

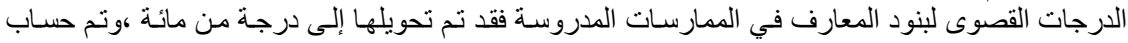

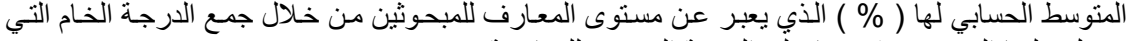

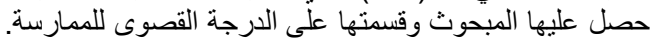

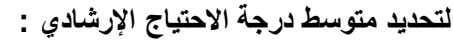

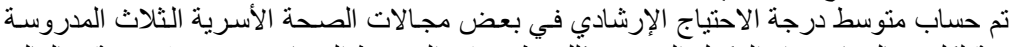

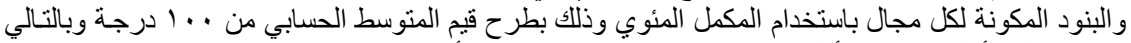

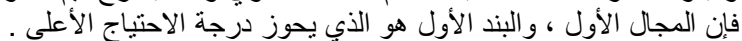

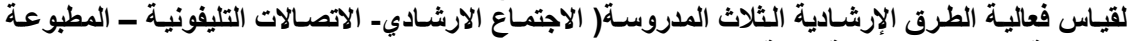
الارشادية) تم تطبيق المعادلة آلاتية: مجموع درجات الاختبار البعدى - مجموع درجات الاختبار القبلي $1 \ldots \times$ مجموع درجات الاختبار البعدي

نسبه التحسن=

سابعا: أدوات التحليل الإحصائي

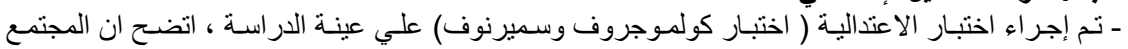

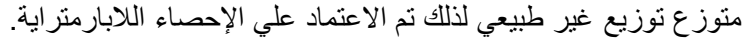

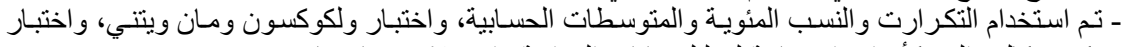

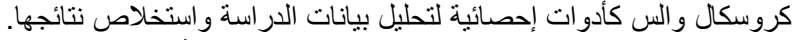
النتائج البحثية

أولاً: مصادر المعلومات التي يستمد منها الريفيات المبحوثات معلوماتهن عن مجالات الصحة الأسرية

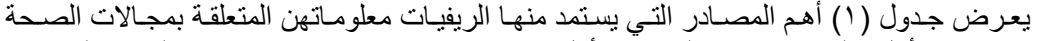

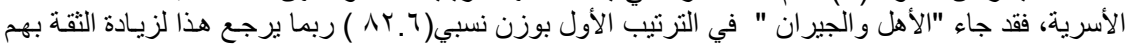

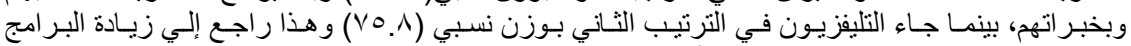

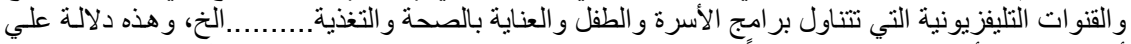

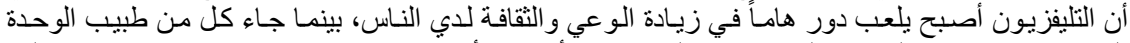

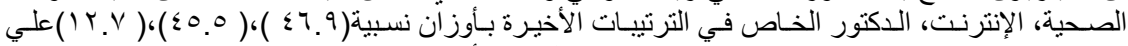

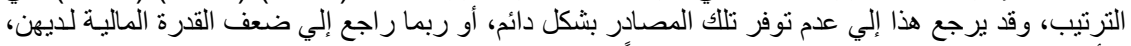

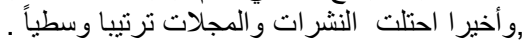


جدول (1) توزيع مصادر المعلومات وفقاً لارجة اعتماد الريفيات المبحوثات عليها

\begin{tabular}{|c|c|c|c|c|c|c|c|c|c|}
\hline \multirow{2}{*}{ الترتيب وفقاً للوزن النسبي } & \multirow{2}{*}{ النسبن } & \multicolumn{2}{|c|}{ ضعيف } & \multicolumn{2}{|c|}{ متوسط } & \multicolumn{2}{|c|}{ عالي } & \multirow{2}{*}{ المصادر } & \multirow[b]{2}{*}{ م } \\
\hline & & $\%$ & العدد & $\%$ & العدد العد & $\%$ & العدد & & \\
\hline 1 & AY. $T$ & YI.A & 7. & A. $\varepsilon$ & rT & 79.1 & 194 & الأهل و الجيران & 1 \\
\hline$\varepsilon$ & $\leqslant 7.9$ & or.A & $1 \leqslant 1$ & $10 . r$ & $\leq r$ & $r \cdot .9$ & 10 & طبيب الوحدة & r \\
\hline r & Vo.A & 19.7 & $0 \leqslant$ & Tr. & 91 & EV.T & Tr. & التليفزيون & $r$ \\
\hline$r$ & $0 \leqslant 0$ & $0 \wedge .0$ & 171 & $19 . r$ & or & TY. & 71 & النشر ات و المجلات & $\varepsilon$ \\
\hline 0 & $\leqslant 0.0$ & $V \wedge . Y$ & Y10 & 7.9 & 19 & $1 \leqslant .9$ & §1 & شبكة الإنترنت & 0 \\
\hline 7 & IY.V & - & - & - & - & IY.V & To & طبيب خاص & 7 \\
\hline
\end{tabular}

ثانياً:الاحتياجات الإرشادية للريفيات المبحوثات في بعض مجالات الصحة الأسرية.

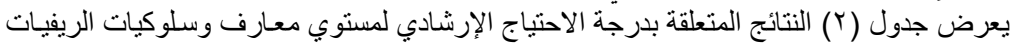

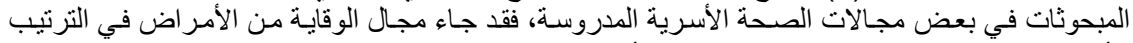

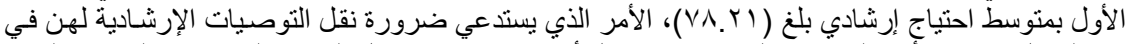

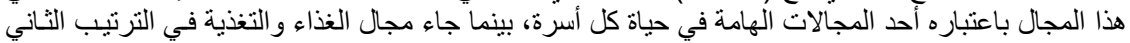

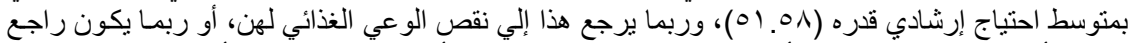

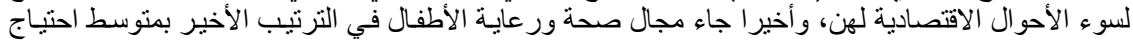

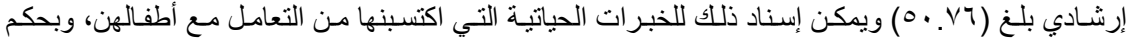

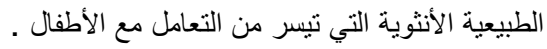

جدول (ץ) توزيع مجالات الصحة الأسرية وفقاً لارجة الاحتيـاج الإرشـادي للريفيات المبحوثنات فيما يتعلق

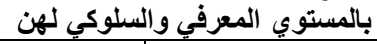

\begin{tabular}{|c|c|c|c|c|}
\hline الاحتياج الإرشادي لدارجة & متوسط درجة الاحتياج & المتوسط الحسابي & المجالات المدروسة & م \\
\hline$r$ & 01.01 & $\leqslant \wedge . \leqslant 1$ & مجال الغذاء و التغذية & $T$ \\
\hline$r$ & $0.1 \mathrm{VT}$ & $\leqslant 9 ., Y \leqslant$ & مجال صحة ور عاية الأطفال & 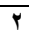 \\
\hline 1 & 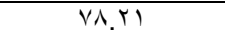 & YI.V9 & لمجال الوقاية من الأمر اض & $r$ \\
\hline
\end{tabular}

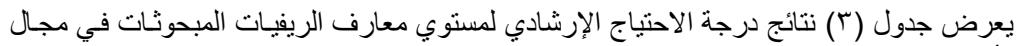

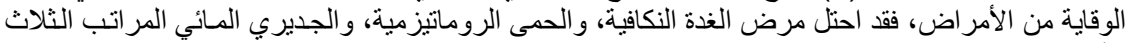

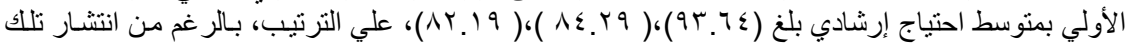

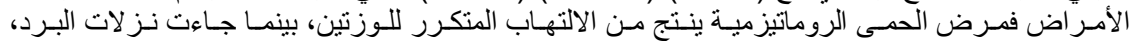

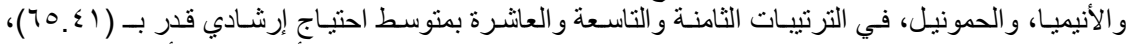

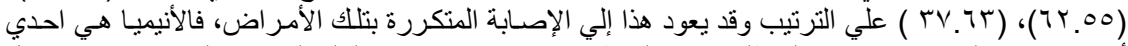

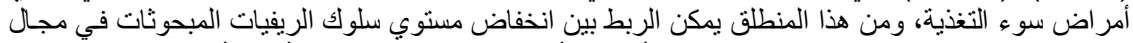

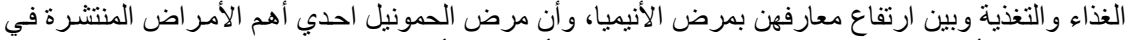

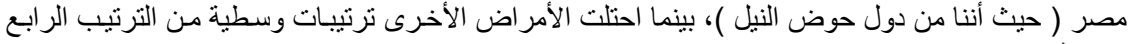
حتى السابع.

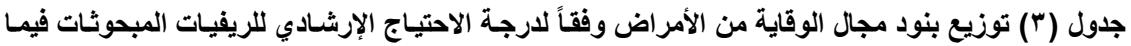
يتعلق بالمستوي المعرفي لهن من الأنة

\begin{tabular}{|c|c|c|c|c|}
\hline & & \multicolumn{3}{|c|}{ ( } \\
\hline | الارجة الإحتياج | الإرشتيب | & متوسط درجة الاحتياج الإرشادي & المتوسط الحسابي & الأمراض المدروسة & p \\
\hline$\wedge$ & $70 . \leqslant 1$ & $r \leqslant .09$ & تزلات البرد & 1 \\
\hline 0 & $V V .97$ & YY.P & |التيفوئيد & r \\
\hline
\end{tabular}


El- Gamal, M. M. A. et al.

\begin{tabular}{|c|c|c|c|c|}
\hline$r$ & Ar. 19 & $\mid V . \Lambda 1$ & |الجديري المائي & $r$ \\
\hline 7 & VV.rI & YY.79 & التهاب ملتحمة العين & $\varepsilon$ \\
\hline 9 & $7 r .00$ & $r v . \leq 0$ & |الأنيميا & $\bullet$ \\
\hline 1 . & rV.Tr & דצ.YT & |الحمونيل & 7 \\
\hline$\varepsilon$ & $1 \cdot .71$ & 19.19 & الأمبيا & V \\
\hline 1 & $94.7 \leq$ & $7 . r 7$ & |الحمي النكافية & $\Lambda$ \\
\hline V & $V Y .+T$ & rV. $9 \leq$ & |التهاب اللوزنين & 9 \\
\hline r & Aะ.Yq & 10.71 & |الحمي الروماتيزمية & 1. \\
\hline \multicolumn{2}{|c|}{$v 0 . \leqslant r$} & $Y \leq .0 \mathrm{~V}$ & \multicolumn{2}{|c|}{ متوسطات معارف مجال الوقاية } \\
\hline
\end{tabular}

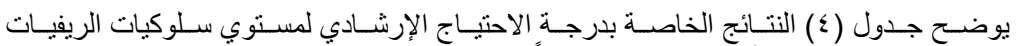

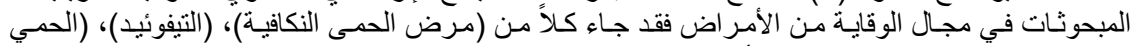

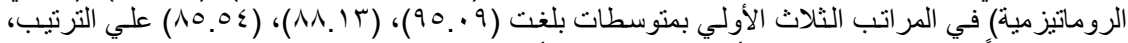

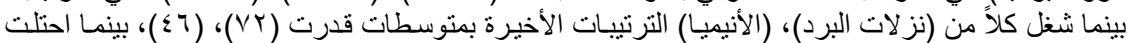

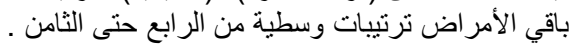

جدول (؛ ) توزيع بنود مجال الوقاية من الأمراض وفقاً لارجة الاحتياج الإرشادي للريفيات المبحوثات فيما

\begin{tabular}{|c|c|c|c|c|}
\hline لارجة الاحتب وفتاً & متوسط درجة الاحتياج & 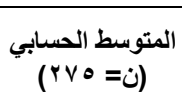 & الأمراض المدروسة & b \\
\hline 9 & VY & rA & انز لات البرد & 1 \\
\hline 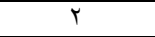 & $1 \Lambda .1 T$ & $11 . \wedge \mathrm{V}$ & |التيفوئيد & $r$ \\
\hline$\varepsilon$ & $\Lambda \Sigma . V \Gamma$ & $10 . \mathrm{KV}$ & |الجديري المائي & $r$ \\
\hline$\wedge$ & $V Y . \cdot Y$ & rY.qA & |التهاب ملتحمة العين & $\varepsilon$ \\
\hline 1. & $\leqslant 7$ & $0 \leq$ & |الأنيميا & 0 \\
\hline 7 & $\wedge \cdot . \wedge 0$ & 19.10 & |الحمونيل & 7 \\
\hline V & V^.TV & r).rT & الأمبيا & $\mathrm{V}$ \\
\hline 1 & 90.9 & $\varepsilon .91$ & |الحمي النكافية & $\Lambda$ \\
\hline 0 & $1 \cdot .9 \leq$ & 19.7 & | التهاب اللوزتين & 9 \\
\hline$r$ & $10.0 \leq$ & $1 \leq . \leq 0$ & |الحمي الروماتيزمية & 1. \\
\hline \multicolumn{2}{|r|}{ 1. .77 } & $19.4 \leq$ & \multicolumn{2}{|l|}{ متوسطات سلوكيات مجال الوقاية من الأمر اض } \\
\hline
\end{tabular}

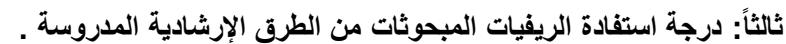

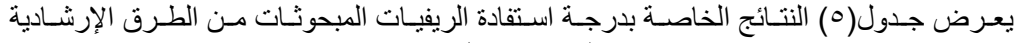

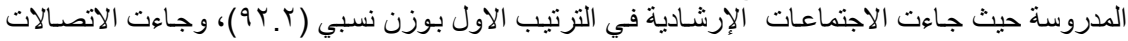

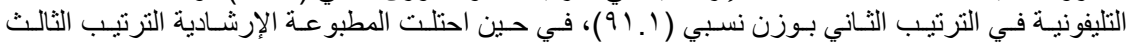

والأخير.

جدول (0) توزيع الطرق الإشادية المدروسة وفقاً لارجة استفادة الريفيات المبحوثات منها

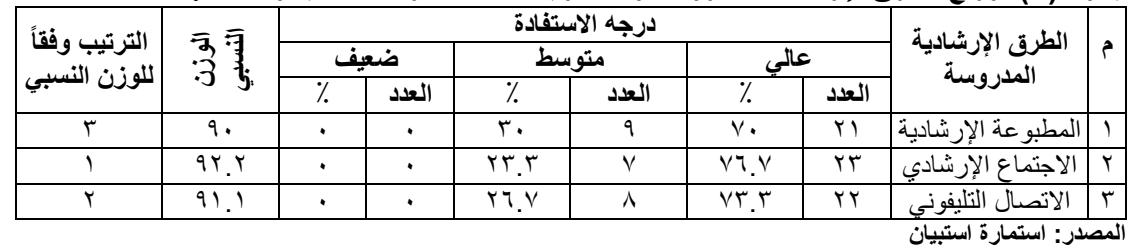




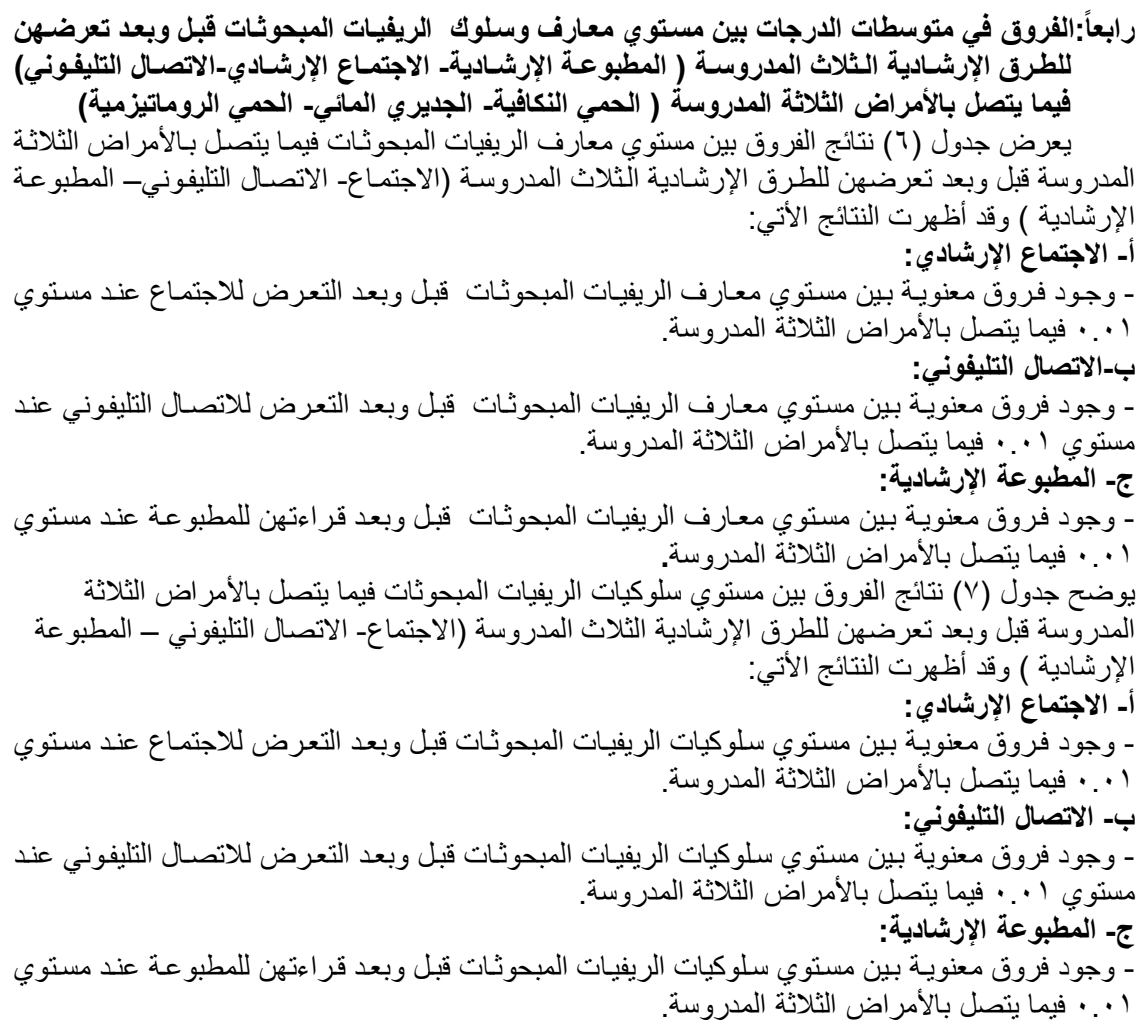


El- Gamal, M. M. A. et al.

V V 
خامساً: الفروق بين الطرق الإرشـادية الثلاث المدروسـة في تحسين المستوي المعرفي والسلوكي للريفيات المبحوثات.

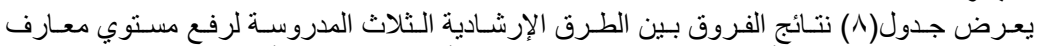

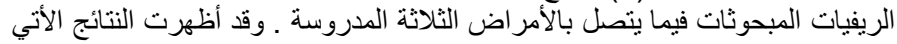

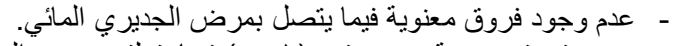

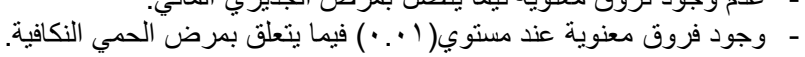

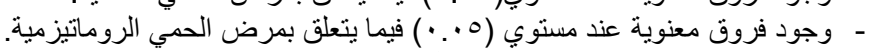

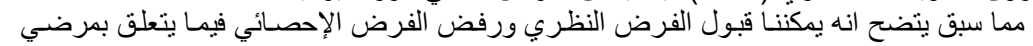

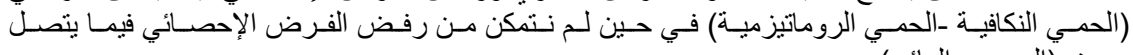
بمرض(الجديري المائي). و هذا يدل علي أن هناك تفاوت بين الطين الطرق الإرشـادية الثناثنة في رفع المستوي المعرفي للريفيات

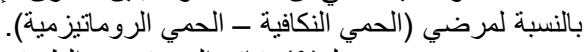

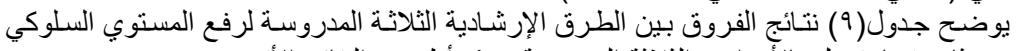

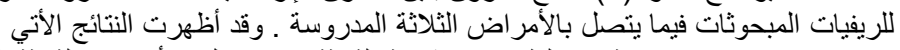

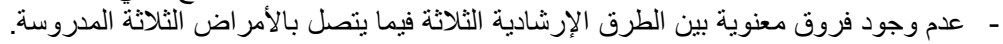

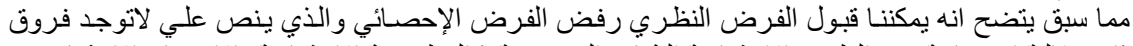

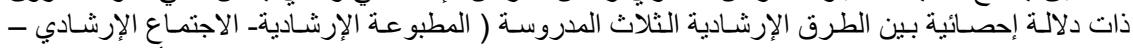

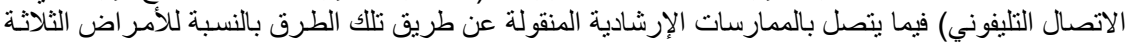

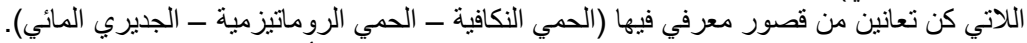

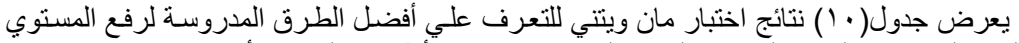

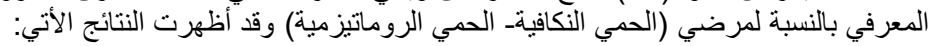

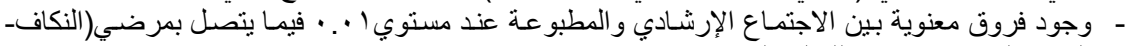

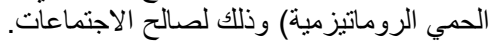

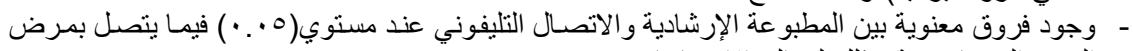

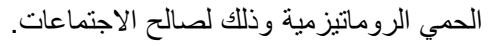

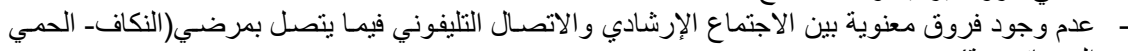
الروماتيزمية).

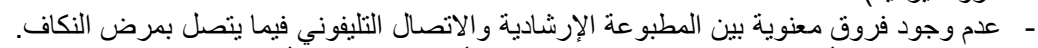

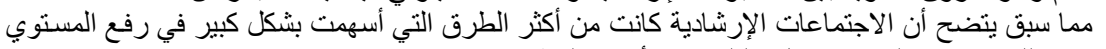

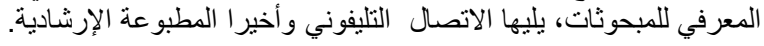

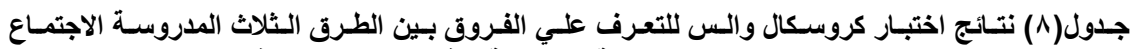

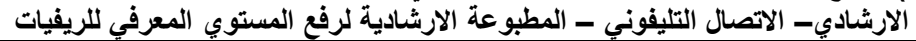

\begin{tabular}{|c|c|c|c|c|c|c|}
\hline \multicolumn{5}{|c|}{ نتائج اختبار كروسكال والس } & \multirow[b]{2}{*}{ الأمراض المدروسة } & \multirow{2}{*}{$p$} \\
\hline مستوية المعنية & كاץ & الإلطبوية & التتليفوني & الإجتماعادي & & \\
\hline${ }^{* *}, \ldots 1$ & V. 9 & r..9. & $\leqslant 0.0$ & $0 \leqslant 0$ & |الحمي النكافية & 1 \\
\hline .1 & r. & $\varepsilon \cdot .7$ & $\varepsilon \varepsilon$ & $01 . \lambda r$ & |الجديري المائي & $r$ \\
\hline$* \cdot . r$ & 7.7 & r. & $\leqslant 9 . r$ & 01.11 & |الحمي الروماتيزمية & $r$ \\
\hline
\end{tabular}

جدول (9) نتائج اختبار كروسكال والس للتعرف علي الفروق بين الطرق الثلاث المدروسة الاجتماعـ- الاتصال التليفوني

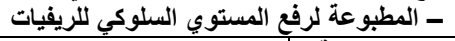
مج| الأمراض المدروسة 
El- Gamal, M. M. A. et al.

\begin{tabular}{|c|c|c|c|c|c|c|}
\hline مستوي المعنوية & كاY & الإرشبوعة المبة & الاتصال التليفوني & الاجتماع الإرشادي & & \\
\hline$\because Y$ & $r .1$ & 01.17 & $\varepsilon r .1$ & $\varepsilon 1 . V V$ & الحمي النكافية & 1 \\
\hline$\because 7$ & .9 & $\varepsilon r . \wedge$ & $\varepsilon \wedge . \vee$ & $\varepsilon \leqslant . \wedge$ & الجديري المائي & r \\
\hline$\cdot . r$ & $r . r$ & $\leq 7.7$ & $\leqslant 9 . \leqslant$ & $\varepsilon \cdot .0$ & الحمي الروماتيزمية & $r$ \\
\hline
\end{tabular}

جدول(· (1) نتائج اختبار مان ويتتي للتعرف علي أفضل الطرق الثلاث المدروسـة في رفع المستوي المعرفي بالنسبة

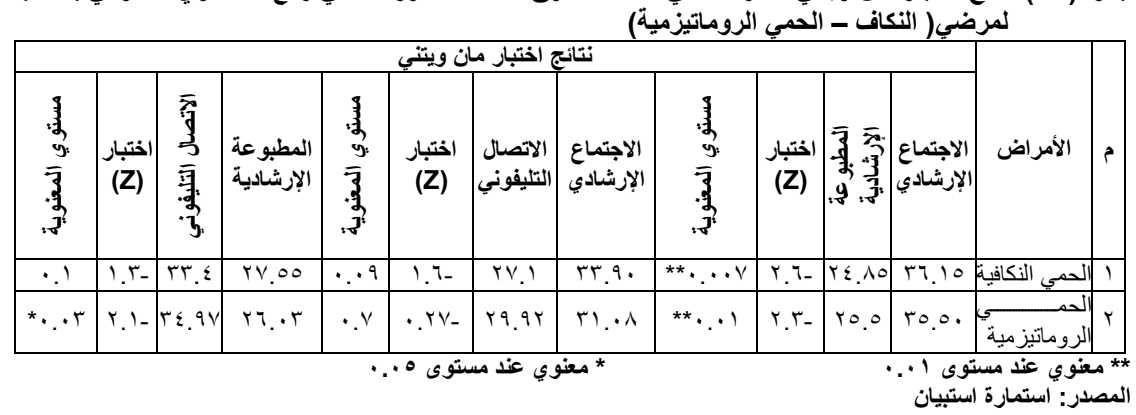

سادساً: : قياس فعالية الطرق الإرشادية الثلاث المدروسة في تحسين المستوي المعرفي والسلوكي للريفيات

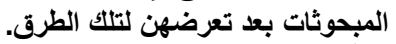

وفقاً للمعادلة التالية تم قياس فعالية الطرق الإرشادية المدروسة.

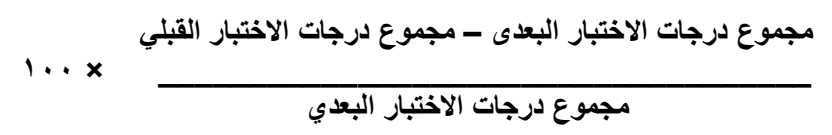

نسبه التحسن=

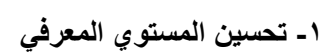

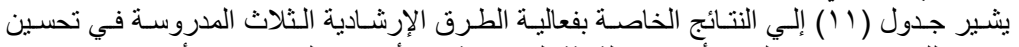
المستوي المعرفي للريفيات فيما يتعلق بالأمر اض الثناثة المدروسة وقد أسفرت النتائج عن الأتي:

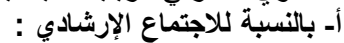

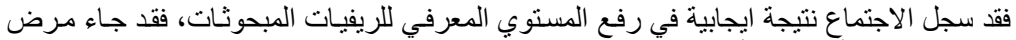

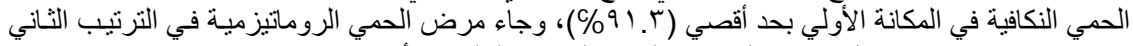

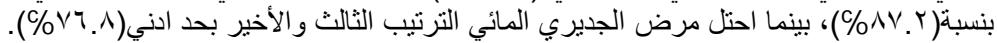

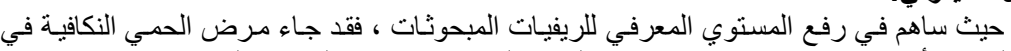

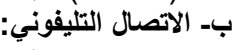

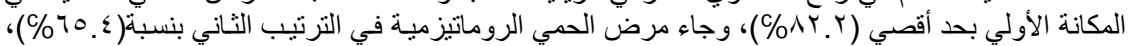

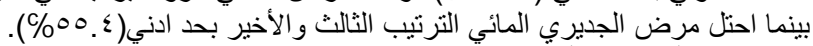

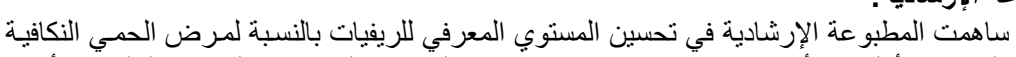

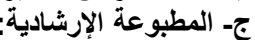

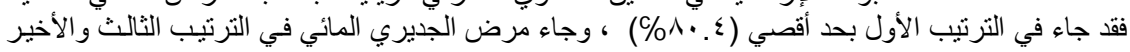

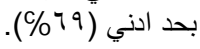

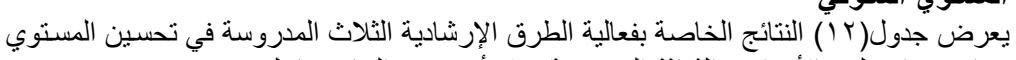

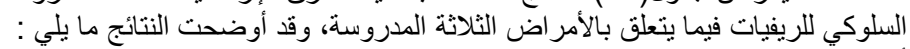

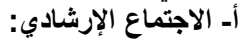




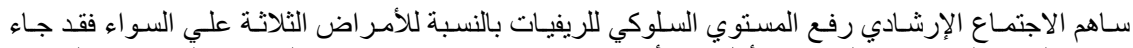

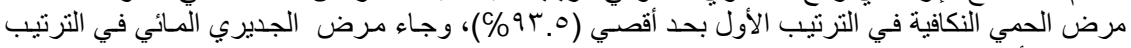

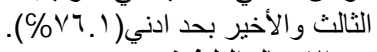

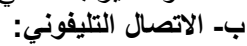

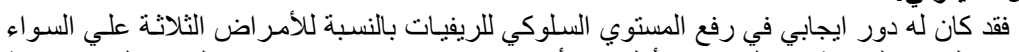

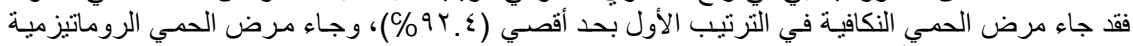

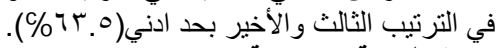
ج- المطبوعة الارشادية:

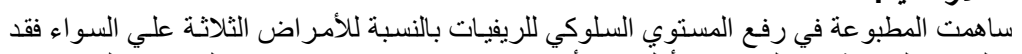

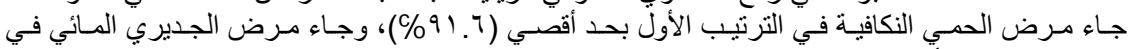

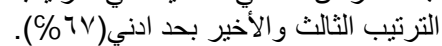

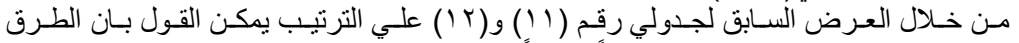

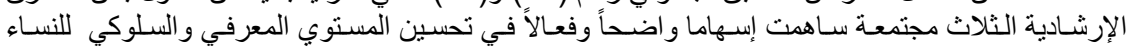
المبحوثات وبدرجة كبيرة نسبيا.

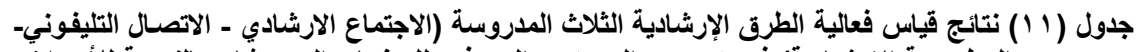

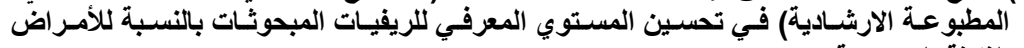

\begin{tabular}{|c|c|c|c|c|c|c|c|c|c|c|c|c|c|}
\hline \multicolumn{4}{|c|}{ المطبو عة الإرشادية } & \multicolumn{4}{|c|}{ الاتصال التليفوني } & \multicolumn{4}{|c|}{ التُلاتَّة المتماروسنة الإرشادي } & \multirow[b]{2}{*}{ الأمر اضة } & 5 \\
\hline 可: & التحعسبة & |لمجارف المجات بعد & قالمعارة الإف قبل & 雪 & التسبة & المجارجات & التمبارت & 可 & |المعرفين & المرجات & |المبارف & & \\
\hline 1 & $\lambda \cdot . \varepsilon$ & AV & IV & 1 & Ar.r & 97 & IV & 1 & $91 . r$ & $1 \cdot \varepsilon$ & 9 & $\begin{array}{l}\bar{y} \\
\text { 司 }\end{array}$ & \\
\hline r & 79 & $9 \mathrm{~V}$ & $r$. & $r$ & $00 . \varepsilon$ & 1.1 & «o & r & $\mathrm{V} .1 .1$ & $1 \cdot 1$ & ro & $\begin{array}{l}\bar{y} \\
\bar{y} \\
\bar{y} \\
\overline{3} \\
\bar{y}\end{array}$ & dr \\
\hline r & V৭.r & 94 & 19 & r & $70 . \varepsilon$ & 11. & ru & r & AV.Y & 11. & $1 \varepsilon$ & 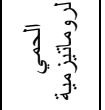 & \\
\hline
\end{tabular}

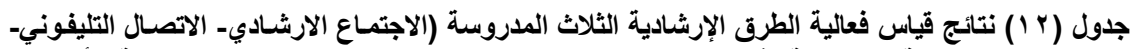

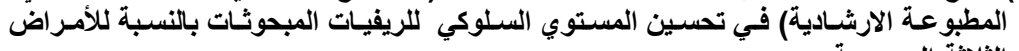

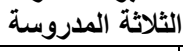

المطبوعة الإرشادية الاتصال التليفوني الاجتماع الإرشادي لادية 
El- Gamal, M. M. A. et al.

\begin{tabular}{|c|c|c|c|c|c|c|c|c|c|c|c|c|}
\hline 帮: & 量 & 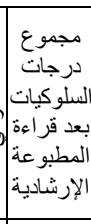 & |السطوكيوتة قلبل جادراءة & 羿 & 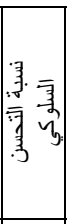 & الملاتلتعبال & 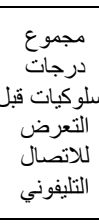 & 型: & 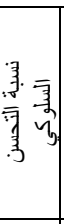 & 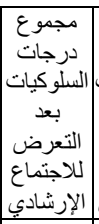 & 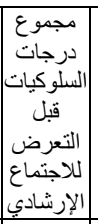 & المدروسة \\
\hline 1 & 91.7 & $\wedge \varepsilon$ & v & 1 & QT. \& & vq & 1 & 1 & $9 \% .0$ & VY & 。 & 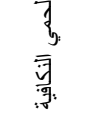 \\
\hline$r$ & IV & 1.5 & $r \varepsilon$ & r & $r^{\lambda} . \lambda$ & 1.9 & $\Gamma \varepsilon$ & $r$ & V4.1 & 1.0 & ro & $\begin{array}{l}\bar{y} \\
\bar{y} \\
\bar{y} \\
\overline{3} \\
\overline{3}\end{array}$ \\
\hline$r$ & va.o & v. & 10 & $r$ & 4.0 & $v \varepsilon$ & rV & $r$ & $\Lambda, . r$ & $1 \xi$ & ir & 可高费 \\
\hline
\end{tabular}

التوصيات

في ضوء ما توصلت إليه الدر اسة من نتائج توصي الدراسة بمجمو عة من التوصيات لعل من أهمها

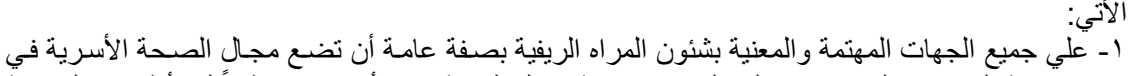

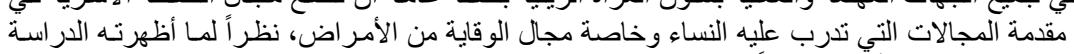

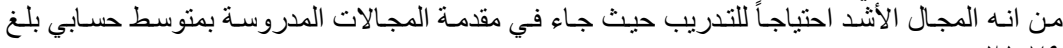
r. . . 9 rـ توصى الدراسة بضرورة تكثيف البرامج الأسرية المقدمة من خلال الإعلام المرئي و المقروء ، و وخاصة في

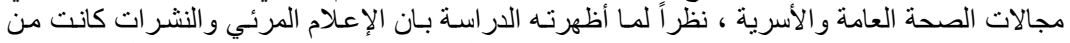

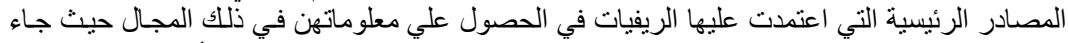

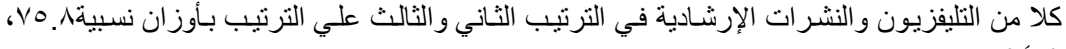
$0 \leqslant 0$

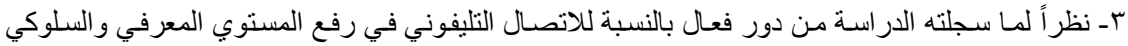

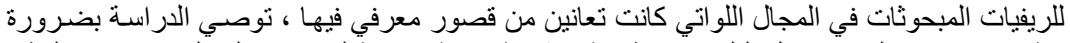

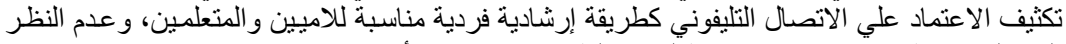

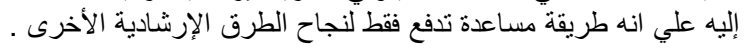

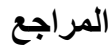

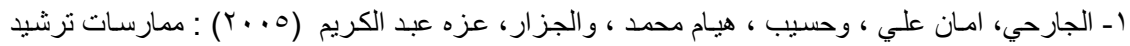

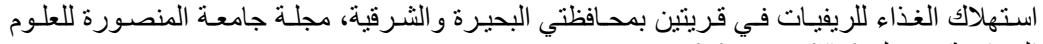

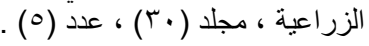




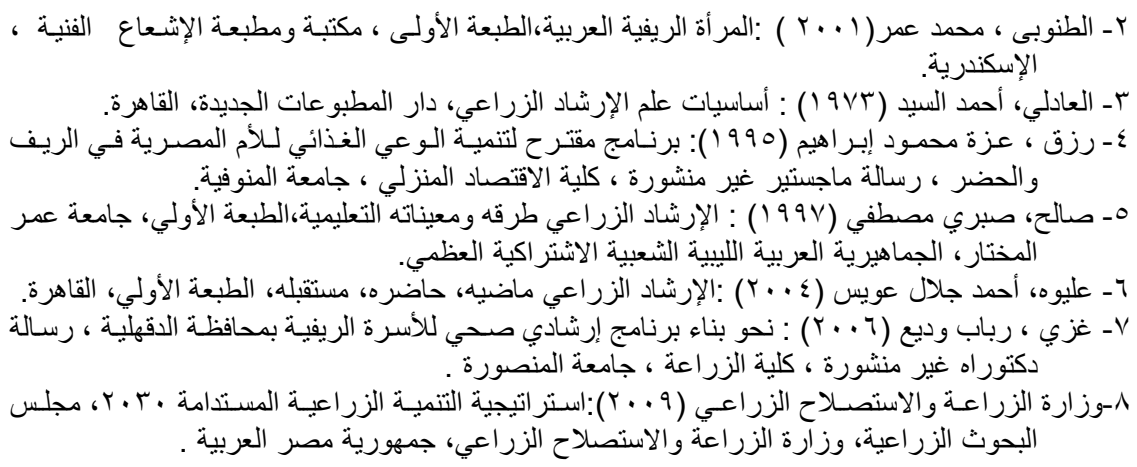

\title{
EFFECTIVENESS OF SOME EXTENSION METHODS IN TRANSFERRING EXTENSION RECOMMENDATIONS FOR RURAL WOMEN IN FAMILY HEALTH AREA “A FIELD STUDY IN DAKAHALIA GOVERNORATE.
}

El- Gamal, M. M. A. ; M. A. M Abd El-Magieed and A. M. A. RAMADAN

Agric. Extension and Rural Society, Fac. of Agric., Mans. Univ.

\begin{abstract}
This study aimed mainly to identify the effectiveness of three different extension methods (meeting, Tel and printed) for improvement of the knowledge and behavioral level of the rural women in some family health areas. The study was carried out at 4 villages in Dakahlia Governorate in two separate periods. The first period conducted from October until December 2011 and included the assessment of the extension needs of a cross sample (275) in the fields of food and diet, children health and disease protection. The shortage of knowledge was recorded in mumps, herbs and rheumatic fever. The second period was from March until December 2012 and included transfer of recommendations about the three previous diseases to 90 women were selected from the aforementioned 275 ones. Thirty women were randomly selected and exposed to the printed method. Sixty women were purposively selected; 30 women were exposed to the telephone method and the other 30 were exposed to the meeting method. After fortnight, the impact of the three methods was measured with the personal interview questionnaire tool. The data were statistically analyzed with frequency, percentages, arithmetic mean, Wilcoxon test, Mann-Whitney test and Kruskal Wallis test.

The study revealed important resulted can be summarized as follow:

1-Disease protection field was in the first rank between the extension needs with complementary percentage (78.21).

2-There are significant differences between the levels of knowledge and behavioral levels of the three diseases $(P<0.01)$ before and after application of the three extension methods.
\end{abstract}




\section{El- Gamal, M. M. A. et al.}

3-The meeting method had contributed in raising of the knowledge level $(91.3 \%)$ and behavioral level $(93.5 \%)$ in mumps disease.

4-The phone method had a significant role in raising the knowledge level $(82.2 \%)$ and the behavioral level $(92.4 \%)$ in mumps disease.

5 -The printed method had contributed in raising of the knowledge level $(80.4 \%)$ and behavioral level $(91.6 \%)$ in mumps disease.

It was recommended that the family health field must be placed on top of the areas which women should be trained. Also, the telephone is considered as an individual extensive method fit illiterate and educated and not only as means of the success of other extension methods.

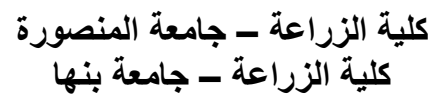

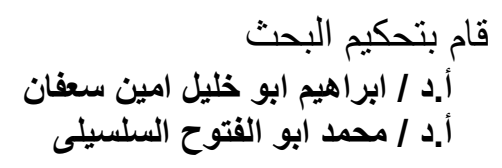


J. Agric. Econom. and Social Sci., Mansoura Univ., Vol.4 (3), March, 2013 
El- Gamal, M. M. A. et al. 


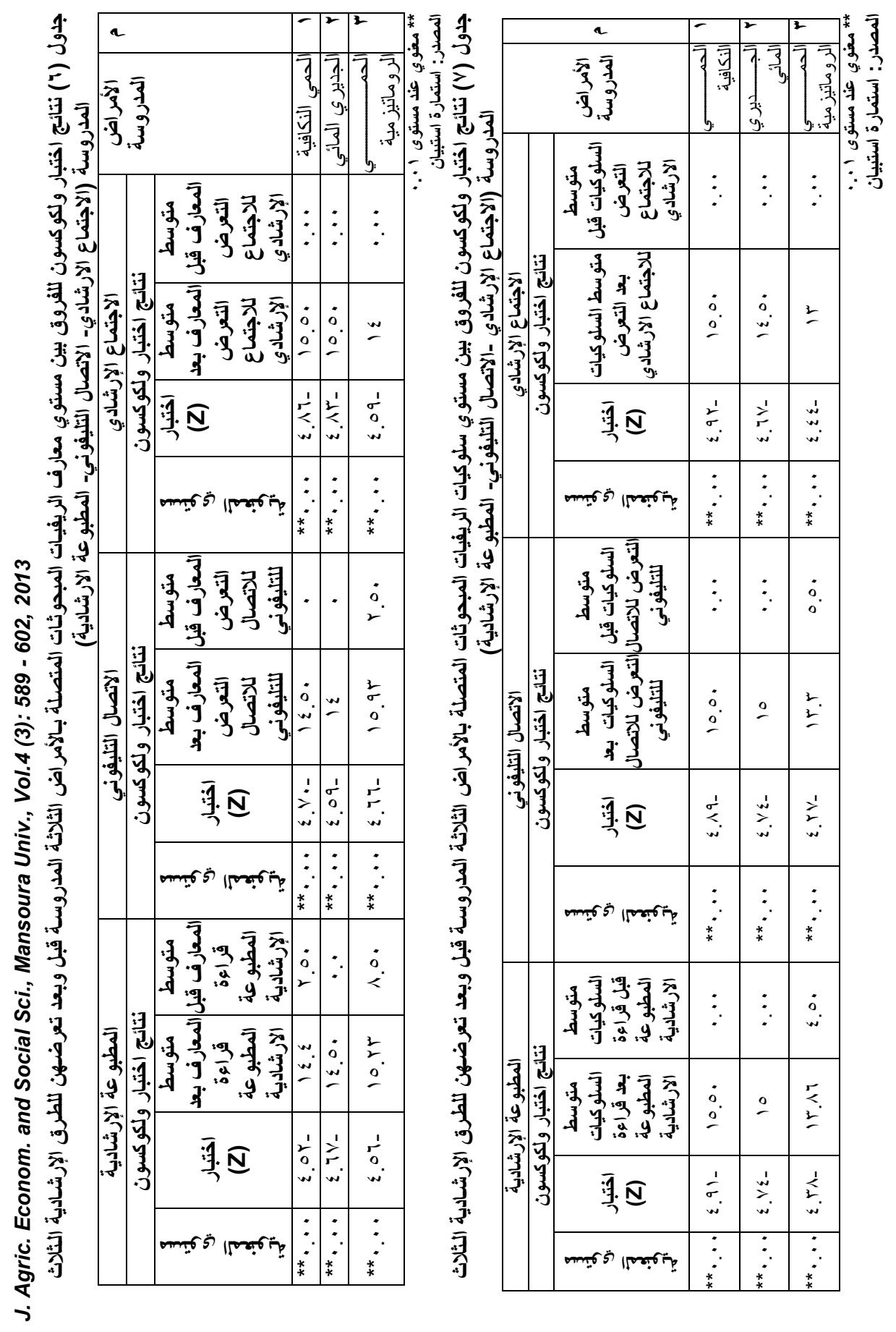

